\title{
Epitaxial decagonal thin films on crystalline substrates
}

\author{
E. J. Widjaja $†$ and L. D. Marks \\ Department of Materials Science and Engineering, Northwestern University, \\ Cook Hall, 2225 N. Campus Drive, Evanston, Illinois 60208-3108, USA
}

[Received 12 June 2002 and accepted 20 September 2002]

\begin{abstract}
$\mathrm{Al}-\mathrm{Cu}-\mathrm{Fe}-\mathrm{Cr}$ quasicrystalline thin films were grown on atomically flat $\mathrm{Al}_{2} \mathrm{O}_{3}$ sapphire (0001) substrates by single-target magnetron sputtering followed by annealing. A decagonal phase with the tenfold axis $\mathrm{A}_{10}$ parallel to the substrate surface normal was observed. The epitaxial decagonal film had two different unique orientations: a twofold $\mathrm{P}$ axis $\mathrm{A}_{2 \mathrm{P}}$ and a twofold $\mathrm{D}$ axis $\mathrm{A}_{2 \mathrm{D}}$ parallel to [1010] of the substrate. These two configurations were explained using a coincidence reciprocal lattice planes model for the interface energy. We show that this classic approach for crystal-crystal epitaxy can be applied to quasicrystal-crystal systems.
\end{abstract}

\section{$\S 1$. InTRODUCTION}

Quasicrystals are orientation-ordered structures with classically forbidden rotation symmetries (e.g. fivefold and tenfold rotation axes) which are incompatible with periodic translational ordering. Quasicrystalline materials exhibit properties that are very different from conventional metallic materials; for reviews of their properties see Archambault and Janot (1997), Jenks and Thiel (1997, 1998), Urban et al. (1997), and Dubois (2000). Industrial applications have employed quasicrystalline materials as a thin coating on conventional crystalline materials (Besser and Eisenhammer 1997, Dubois 2000). Therefore, it is important to establish the geometric relationship between quasicrystal coatings and crystal substrates.

Research in this area had been limited to establishing the relationship between quasicrystal single crystals to crystalline phases induced by ion sputtering (Zhang and Geng 1992, Zhuang et al. 1993, Shen et al. 1998) and quasicrystal precipitates to their crystalline matrices due to ion implantation (Zhang et al. 1997). These may impose restrictions on the geometric orientation because the phases appear as a result of local rearrangement or displacement of atoms due to high-energy particles. More recently, there have been studies on the geometric relationship between crystalline thin films on quasicrystal substrates by physical vapour deposition. Shimoda et al. (2000) attempted to grow a Au thin film (about $0.19 \mathrm{~nm}$ ) on decagonal Al-Ni-Co and reported the orientation of the alloyed $\mathrm{AuAl}_{2}$ layer with respect to the substrates. Bolliger et al. (2001) reported epitaxial growth of an Al film (less than $8 \mathrm{~nm}$ ) on the fivefold-symmetric surface of icosahedral Al-Pd-Mn; however, the stability was limited to $50^{\circ} \mathrm{C}$ owing to diffusion of $\mathrm{Al}$ into the sample. Li et al. (1997) reported

\footnotetext{
†Email: e-widjaja@northwestern.edu.

Philosophical Magazine Letters ISSN 0950-0839 print/ISSN 1362-3036 online (C) 2003 Taylor \& Francis Ltd http://www.tandf.co.uk/journals DOI: $10.1080 / 0950083021000045760$
} 
growth of fully oriented $\mathrm{Al}-\mathrm{Cu}-\mathrm{Co}$ decagonal films on crystalline substrates using Xray diffraction but was unable to study the geometric orientations at the interface.

In none of the work to date has there been any attempt to explain the observed orientational relationship. In addition, it is not clear in any of the prior work whether true thermodynamic equilibrium of the quasicrystal-substrate interface has been achieved. Here we report the geometric relationship of $\mathrm{Al}-\mathrm{Cu}-\mathrm{Fe}-\mathrm{Cr}$ decagonal thin films on crystalline substrates produced by magnetron sputtering and annealed to equilibration. We show that the observed orientations follow the same rules as governing those for crystal-crystal interfaces.

\section{§ 2. EXPERIMENTAL DETAILS}

Aluminium oxide $\left(\mathrm{Al}_{2} \mathrm{O}_{3}\right)$ (sapphire) (0001) substrates were prepared using standard transmission electron microscopy (TEM) sample preparation techniques: cutting to a $3 \mathrm{~mm}$ disc, thinning, mechanical dimpling, followed by $\mathrm{Ar}^{+}$-ion milling to perforation. Subsequently the substrates were annealed in air at $1400^{\circ} \mathrm{C}$ for $2-4 \mathrm{~h}$ to allow recovery and recrystallization from defects introduced during preparation (Kim and Hsu 1992, Susnitzky and Carter 1992).

Substrates were inserted into the sample preparation, evaluation, analysis and reaction (SPEAR) system which allows sample manipulation and transfer under ultrahigh vacuum. The system includes a magnetron sputtering chamber for thinfilm growth, a Hitachi ultrahigh vacuum $\mathrm{H}-9000$ transmission electron microscope and a Phi X-ray photoelectron spectroscope. Details of the system (Collazo-Davila et al. 1995) and preliminary in-situ results on the growth (Widjaja and Marks 2002) have been published elsewhere.

After insertion into the ultrahigh-vacuum chamber, samples were evaluated using X-ray photoelectron spectroscopy (XPS) and TEM to ensure surface cleanliness and to document the surface morphology prior to deposition. XPS studies show clean $\mathrm{Al}_{2} \mathrm{O}_{3}$ surfaces and TEM studies reveal the presence of atomically flat terraces $100-150 \mathrm{~nm}$ wide.

Thin films were deposited in a magnetron sputtering chamber with a base pressure of $6.7 \times 10^{-8} \mathrm{~Pa}$. Deposition was carried out in $\mathrm{Ar}(99.998 \%$ purity) at $3.7 \times 10^{-1} \mathrm{~Pa}, 150 \mathrm{~W}$ and no substrate bias. The deposition rate at room temperature was determined to be approximately $13 \mathrm{~nm} \mathrm{~min}^{-1}$. The average compositions of the films were $\mathrm{Al}_{86.3 \pm 4.4} \mathrm{Cu}_{3.9 \pm 0.7} \mathrm{Fe}_{5.4 \pm 0.4} \mathrm{Cr}_{4.4 \pm 0.5}$ and $\mathrm{Al}_{82.5 \pm 4.3} \mathrm{Cu}_{4.0 \pm 0.5} \mathrm{Fe}_{7.9 \pm 0.7} \mathrm{Cr}_{5.5 \pm 0.3}$ as measured by in-situ XPS and ex-situ Energy-dispersive X-ray spectroscopy (EDXS) respectively (calibrated via atomic emission spectroscopy). Measurements by EDXS of the composition of single grains of the decagonal phase gave a composition of $\mathrm{Al}_{78.3 \pm 4.5} \mathrm{Cu}_{7.6 \pm 1.4} \mathrm{Fe}_{7.5 \pm 1.9} \mathrm{Cr}_{6.6 \pm 1.0}$. As a consequence, the overall film was not phase pure, but this is not relevant for the results described herein.

The samples were taken out from the ultrahigh-vacuum chamber and put in a flowing Ar tube furnace for annealing. The annealing treatment proceeded in two steps: preheating at $575^{\circ} \mathrm{C}$ for $22 \mathrm{~h}$ and subsequent heating at $800^{\circ} \mathrm{C}$ for $2 \mathrm{~h}$. The samples were then brought to room temperature with a cooling rate of less than $4^{\circ} \mathrm{C} \mathrm{min}^{-1}$. Studies on thin films annealed at $300-400^{\circ} \mathrm{C}$ did not show any specific orientations between the decagonal films and the atomically flat crystalline substrates (Widjaja and Marks 2002). The preheating temperature was chosen to allow reorientation of grains because, at this temperature, solid diffusion is enhanced (Widjaja and Marks 2002). Additional heating at $800^{\circ} \mathrm{C}$ was intended to equilibrate the samples as observed in multilayer post-annealing of $\mathrm{Al}-\mathrm{Cu}-\mathrm{Co}$ decagonal mat- 
erial (Li et al. 1997). The annealed samples were studied using a Hitachi H-8100 transmission electron microscope.

Samples for diffraction intensity measurement were deposited on sodium chloride crystals at room temperature and annealed in flowing Ar to form the decagonal phase. The substrates were removed by dissolving in water to acquire free-standing thin films. These were then suspended on holey-C films in Ni grids and studied using a JEOL-3010 transmission electron microscope. Diffraction patterns were recorded digitally using Digital Micrograph 3.00 and were processed using Semper software; the diffraction intensity were measured using a cross-correlation method $(\mathrm{Xu}$ et al. 1994).

\section{§ 3. RESULTS}

Selected-area diffraction patterns on the annealed samples as shown in figure 1 correspond to a decagonal diffraction pattern superimposed on the substrate diffraction pattern. The decagonal phases in the thin films have the tenfold axis oriented parallel to the substrate surface normal, $\mathrm{A}_{10} / / \mathrm{Al}_{2} \mathrm{O}_{3}[0001]$.

The diffractions pattern of the decagonal phase along the tenfold direction and the sapphire substrate show tenfold symmetry (equivalent to $36^{\circ}$ rotation) and threefold symmetry (equivalent to $120^{\circ}$ rotation). Therefore the unique orientation of both diffraction patterns is limited to $12^{\circ}$ of rotation. Only two unique relative orientations were observed: $\mathrm{A}_{2 \mathrm{D}} / / \mathrm{Al}_{2} \mathrm{O}_{3}[10 \overline{1} 0]$ and $\mathrm{A}_{2 \mathrm{P}} / / \mathrm{Al}_{2} \mathrm{O}_{3}[10 \overline{1} 0]$, where $\mathrm{A}_{2 \mathrm{D}}$ and $\mathrm{A}_{2 \mathrm{P}}$ represent the two types of twofold axis in the decagonal phase.

\section{§ 4. Analysis}

A common approach for describing the observed two unique orientations is symmetry and alignment of rotation axis (Zhang and Geng 1992, Zhang et al. 1997, Shen et al. 1998, Bolliger et al. 2001). In the configuration observed in figure 1 , there are common symmetry elements, namely mirror planes, which compose the subgroup common to both structures, and alignment of the twofold decagonal axis with the twofold axis of the sapphire.

However, while this is a viable way of describing the orientation relationship, it does not answer the fundamental question: the energetics. Interface energies between two crystalline materials are relatively well understood, and there are three commonly used models: the coincident-site lattice model (Grimmer et al. 1984, Grimmer 1989, Wolf and Yip 1992, Sutton and Baluffi 1995), coincidence of reciprocal-lattice planes (CRLP) (Fletcher and Adamson 1966, Ikuhara and Pirouz 1996, Stemmer et al. 1996) and the $d$-spacing concept (Sutton 1992, Wolf and Yip 1992, Sutton and Baluffi 1995). Of these the original CRLP model can be applied to quasicrystals, with one important extension that appears to have been omitted to date in the literature.

Following Fletcher and Lodge (1975), the energy of a boundary can be written as a combination of two terms: an interface energy $E_{\mathrm{i}}$ and an elastic strain energy $E_{\mathrm{s}}$. With a displacement of

$$
F(\mathbf{R})=-\sum_{\boldsymbol{\kappa}}^{+} 2\left[D_{\boldsymbol{\kappa}} \sin (\boldsymbol{\kappa} \cdot \mathbf{R})+C_{\boldsymbol{\kappa}} \cos (\boldsymbol{\kappa} \cdot \mathbf{R})\right]-C_{0}
$$

They wrote the interface energy in the very general form 

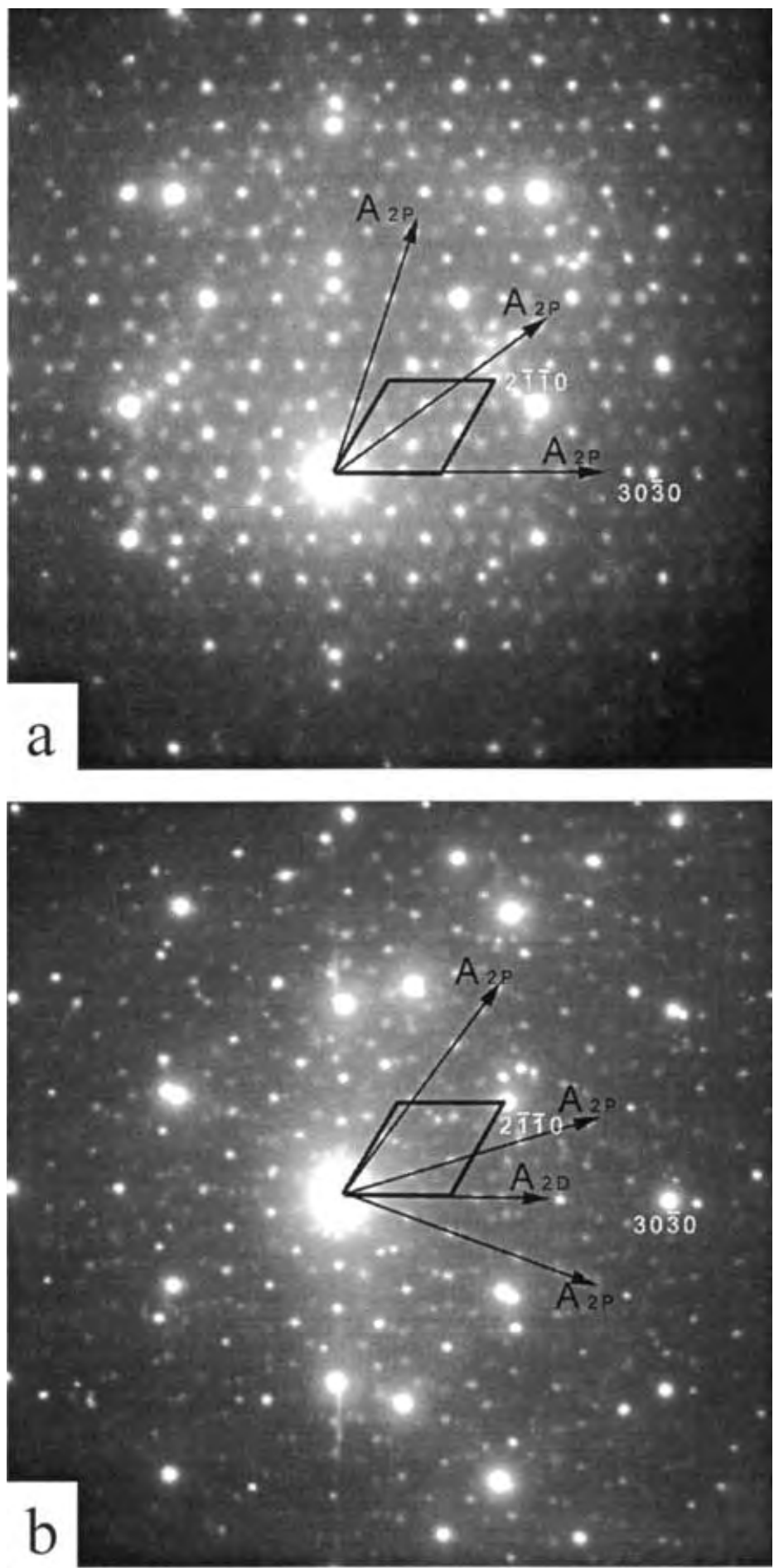

Figure 1. Superimposed diffraction patterns from decagonal quasicrystals on a sapphire substrate showing $\mathrm{A}_{10} / / \mathrm{Al}_{2} \mathrm{O}_{3}[0001]$ : (a) $\mathrm{A}_{2 \mathrm{P}} / / \mathrm{Al}_{2} \mathrm{O}_{3}[10 \overline{1} 0] ;$ (b) $\mathrm{A}_{2 \mathrm{D}} / / \mathrm{Al}_{2} \mathrm{O}_{3}[10 \overline{10}]$. The two unique orientations are related by a $6^{\circ}$ rotation of the decagonal phase along the tenfold axis with respect to the crystalline substrate. 


$$
\begin{aligned}
E_{\mathrm{i}}= & \frac{1}{2 \pi A A^{\prime}} \sum_{\mathbf{k}} v(\mathbf{k}) \exp (\mathrm{ik} \cdot \mathbf{B}) x\left(\prod_{\boldsymbol{\kappa}} J_{0}\left(2 \mathbf{k} \cdot \mathbf{D}_{\boldsymbol{\kappa}}\right) J_{0}\left(2 \mathbf{k} \cdot \mathbf{C}_{\boldsymbol{\kappa}}\right)\right) \\
& \times\left(\prod_{\boldsymbol{\kappa}} J_{0}\left(2 \mathbf{k} \cdot \mathbf{D}_{\boldsymbol{\kappa}}^{\prime}\right) J_{0}\left(2 \mathbf{k} \cdot \mathbf{C}_{\boldsymbol{\kappa}}^{\prime}\right)\right) \\
& \times\left[\delta_{\mathbf{k}, \mathbf{q}} \delta_{\mathbf{k}, \mathbf{q}^{\prime}}+\sum_{n=1}^{\infty} \sum_{\boldsymbol{\kappa}}+\left(\frac{J_{n}\left(2 \mathbf{k} \cdot \mathbf{D}_{\boldsymbol{\kappa}}\right)}{J_{0}\left(2 \mathbf{k} \cdot \mathbf{D}_{\boldsymbol{\kappa}}\right)}+\mathrm{i}^{n} \frac{J_{n}\left(2 \mathbf{k} \cdot \mathbf{C}_{\boldsymbol{\kappa}}\right)}{J_{0}\left(2 \mathbf{k} \cdot \mathbf{C}_{\boldsymbol{\kappa}}\right)}\right) \delta_{\mathbf{q}+\mathbf{n} \boldsymbol{\kappa}, \mathbf{q}^{\prime}} \delta_{\mathbf{k}, \mathbf{q}^{\prime}}\right. \\
& \left.+\sum_{n=1}^{\infty} \sum_{\boldsymbol{\kappa}}+\left(\frac{J_{n}\left(2 \mathbf{k} \cdot \mathbf{D}_{\boldsymbol{\kappa}}^{\prime}\right)}{J_{0}\left(2 \mathbf{k} \cdot \mathbf{D}_{\boldsymbol{\kappa}}^{\prime}\right)}+\mathrm{i}^{n} \frac{J_{n}\left(2 \mathbf{k} \cdot \mathbf{C}_{\boldsymbol{\kappa}}^{\prime}\right)}{J_{0}\left(2 \mathbf{k} \cdot \mathbf{C}_{\boldsymbol{\kappa}}^{\prime}\right)}\right) \delta_{\mathbf{q}+\mathbf{n} \boldsymbol{\kappa}, \mathbf{q}^{\prime}} \delta_{\mathbf{k}, \mathbf{q}}+\cdots\right]
\end{aligned}
$$

where $A$ and $A^{\prime}$ are the areas of interface unit cells, $v(\mathbf{k})$ is Fourier transform of the atomic potential, $\int v(\mathbf{r}) \exp (-\mathrm{ik} \cdot \mathbf{r}) \mathrm{d} \mathbf{r}, \mathbf{B}$ is the relative translation of the two lattices which have $\mathbf{q}$ and $\mathbf{q}^{\prime}$ reciprocal-lattice vectors, $J_{0}$ and $J_{n}$ are Bessel functions and $\boldsymbol{\kappa}$ is a vector corresponding to allowed distortion components.

In the original derivation, only a simple basis for the unit cell was employed, and the delta functions arose via a sum over all atoms in the interface, that is

$$
\sum_{\mathbf{R}} \exp (\mathrm{i} \mathbf{k} \cdot \mathbf{R})=N \delta_{\mathbf{k}, \mathbf{q}}
$$

for $N$ atoms. This is not correct in general, and the equation has to be modified by replacing $\delta_{\mathbf{k}, \mathbf{q}} \delta_{\mathbf{k}, \mathbf{q}^{\prime}}$ with $U(\mathbf{q}) U\left(\mathbf{q}^{\prime}\right) \delta_{\mathbf{k}, \mathbf{q}} \delta_{\mathbf{k}, \mathbf{q}}$ where

$$
U(\mathbf{q})=\sum_{\mathbf{R}} \exp (\mathrm{iq} \cdot \mathbf{R}),
$$

with a sum over the basis of the unit cell. This term, which by analogy to crystallographic direct methods where it also arises, we shall call a unitary structure factor. We note that it is statistically related to $I(\mathbf{q})$, kinematic electron (or X-ray) diffraction intensities (Giacovazzo 1998) for the outermost layer of the material on each side of the interface by

$$
U(\mathbf{q}) \propto \frac{[I(\mathbf{q})]^{1 / 2}}{\langle f(\mathbf{q})\rangle}
$$

where $\langle f(\mathbf{q})\rangle$ is the expectation value of the single-atom scattering factors. For thin regions there will be some dynamic diffraction, but this relationship should remain approximately correct. Even though a quasicrystal does not have a three-dimensional lattice, it does form discrete reciprocal-lattice points so this form can still be used. A more detailed analysis, including use of traction matching (as against the variational approach used originally) will be presented elsewhere. Here we shall follow the original work and exploit a simple first-order approach, which gives a total energy of form

$$
E=E_{0}-\sum_{\boldsymbol{\kappa}} \frac{\left[U(\mathbf{q}) v_{0}(\mathbf{q})\right]^{2}}{\boldsymbol{\kappa}},
$$

where $\boldsymbol{\kappa}$ is the vector joining two diffraction spots from the bicrystal and $v_{0}(\mathbf{q})$ is the atomic interaction potential which is at the present time unknown for the quasicrystal-crystal interface. We took $v_{0}(\mathbf{q})$ to be unity, which will introduce some error but should not substantively alter the results. 
A schematic reciprocal-lattice plane is shown in figure 2. We included the (1010)type diffraction spots of alumina (corundum structure) which is forbidden in the bulk but allowed at a surface or interface. The values of $U(\mathbf{q})$ were taken as $[I(\mathbf{q})]^{1 / 2}$ for the quasicrystal and as unity for the alumina substrate. Since $E \propto-1 / \boldsymbol{\kappa}$, the spots with small $\boldsymbol{\kappa}$ values dominating the energy term. Therefore, only the set of
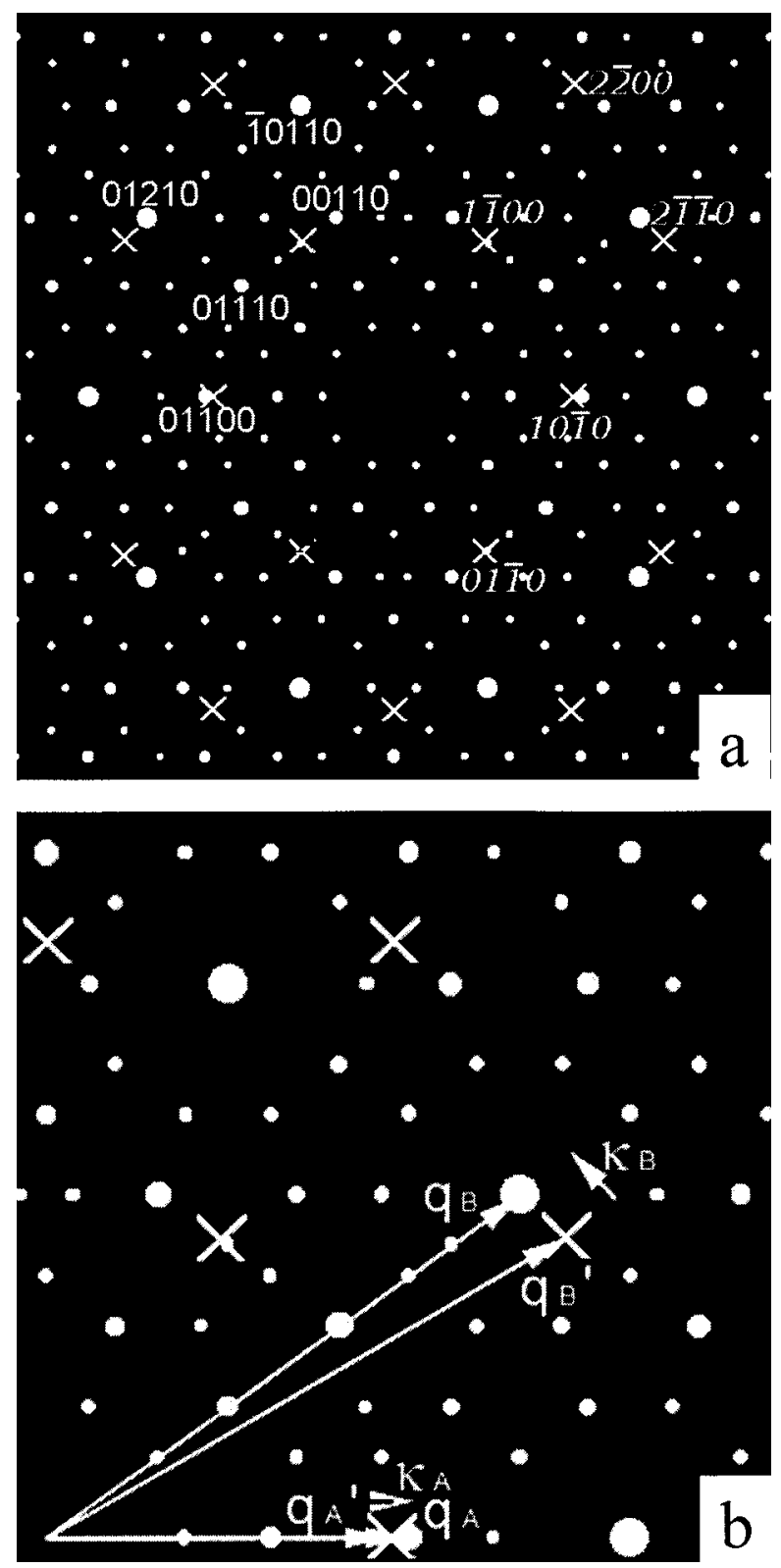

Figure 2. Schematic reciprocal-lattice planes of decagonal quasicrystal and alumina crystal: (a) decagonal diffraction spots (white full circles) and alumina diffraction spots (white crosses); (b) decagonal vectors $\mathbf{q}_{\mathrm{A}}$ and $\mathbf{q}_{\mathrm{B}}$ and alumina vectors $\left(\mathbf{q}_{\mathrm{A}}^{\prime}\right.$ and $\mathbf{q}_{\mathrm{B}}^{\prime}$ ) in the reciprocal-lattice and their corresponding $\boldsymbol{\kappa}$ vectors $\boldsymbol{\kappa}_{\mathrm{A}}$ and $\boldsymbol{\kappa}_{\mathrm{B}}$. 

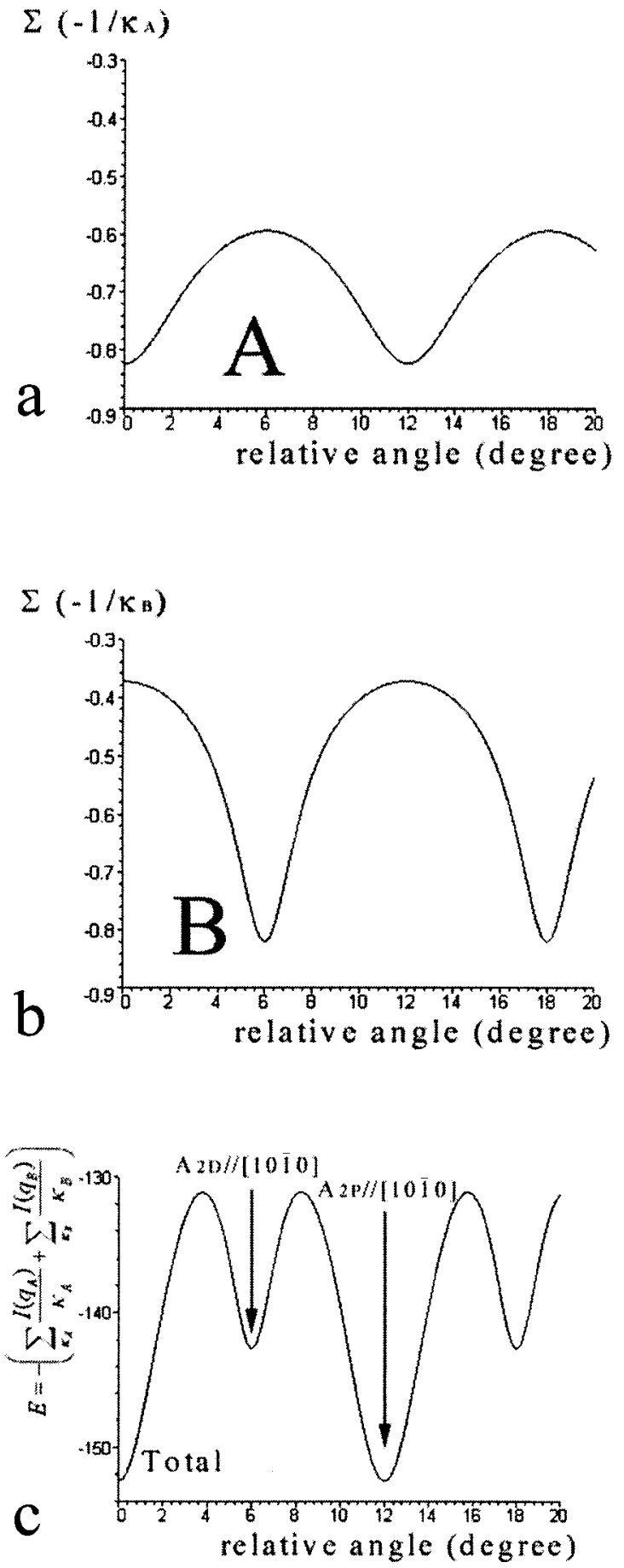

Figure 3. Energy calculation: (a) $E=-1 / \boldsymbol{\kappa}_{\mathrm{A}}$ for set A; (b) $E=-1 / \boldsymbol{\kappa}_{\mathrm{B}}$ for set B, (c) total energy $E=-\left\{\sum_{\kappa_{\mathrm{A}}}\left[I\left(\mathbf{q}_{\mathrm{A}}\right) / \boldsymbol{\kappa}_{\mathrm{A}}\right]+\sum_{\boldsymbol{\kappa}_{\mathrm{B}}}\left[I\left(\mathbf{q}_{\mathrm{B}}\right) / \boldsymbol{\kappa}_{\mathrm{B}}\right]\right\}$ showing two minima corresponding to $\mathrm{A}_{2 \mathrm{P}} / / \mathrm{Al}_{2} \mathrm{O}_{3}[10 \overline{1} 0]$ and $\mathrm{A}_{2 \mathrm{D}} / / \mathrm{Al}_{2} \mathrm{O}_{3}[10 \overline{1} 0]$. The graph shows the $12^{\circ}$ rotation periodicity. 
spots that are closest to $(10 \overline{1} 0)$ and $(1 \overline{2} 10)$, set A for the (11100) spot and set B corresponding to the (12210) reflection, needed to be considered in the calculation.

Reciprocal spacings of (10 $\overline{1} 0)$ and (1210) of $\mathrm{Al}_{2} \mathrm{O}_{3}$ and (11100) and (12210) of the decagonal were calculated and measured to be $2.4243 \mathrm{~nm}^{-1}, 4.1990 \mathrm{~nm}^{-1}$, $2.5399 \pm 0.0219 \mathrm{~nm}^{-1}$ and $4.1068 \pm 0.0319 \mathrm{~nm}^{-1}$ respectively. The measured intensity ratio $I_{\mathrm{A}} / I_{\mathrm{B}}$ was $2.7 \pm 0.6$.

Starting with the configuration where $A_{2 P}$ is aligned with [1010], and using this as the rotational origin of $0^{\circ}$, the summation of $-I(\mathbf{q}) / \kappa$ for the spots from different sets,

$$
E \approx-\left(\sum_{\boldsymbol{\kappa}_{\mathrm{A}}} \frac{I\left(\mathbf{q}_{\mathrm{A}}\right)}{\boldsymbol{\kappa}_{\mathrm{A}}}+\sum_{\boldsymbol{\kappa}_{\mathrm{B}}} \frac{I\left(\mathbf{q}_{\mathrm{B}}\right)}{\boldsymbol{\kappa}_{\mathrm{B}}}\right),
$$

is plotted in figure 3. There are energy minima, at $0^{\circ}\left(\mathrm{A}_{2 \mathrm{P}} / /[10 \overline{1} 0]\right)$ and $6^{\circ}$ $\left(\mathrm{A}_{2 \mathrm{P}} / /[1 \overline{2} 10]\right)$. These minima are equivalent to $\mathrm{A}_{2 \mathrm{P}} / /[10 \overline{1} 0]$ and $\mathrm{A}_{2 \mathrm{D}} / /[10 \overline{1} 0]$, which are the observed orientations.

\section{$\S 5$. Discussion}

The results reported herein indicate that quasicrystalline materials can have specific orientational relationships in interfaces to crystals, and that these obey very similar rules to those that govern crystalline interfaces. In real space it is hard to model this (except via some large approximant to the quasicrystal), but the first-order approach is viable and should be completely general in reciprocal space. The $U(\mathbf{q})$ term that should be included will also weight the analysis towards orientations with more atoms aligned, and may be important in general although often the $1 / \boldsymbol{\kappa}$ term will dominate. (More accurate analyses require expansions to higher order, which may not be so tractable.)

We would presume that the interface is relaxed by misfit dislocations, similar to crystal-crystal interfaces. The exact form of these is an interesting question. With a softer material connected to a quasicrystal, we would expect a quasiperiodic array of dislocations. While there is a suggestion of this in the literature (Zhang and Geng 1992, Zhuang et al. 1993), a complete analysis does not appear to exist as yet.

\section{$\S 6$. CONCLUSIONS}

$\mathrm{Al}-\mathrm{Cu}-\mathrm{Fe}-\mathrm{Cr}$ quasicrystalline thin films were grown on atomically flat $\mathrm{Al}_{2} \mathrm{O}_{3}$ (sapphire) (0001) substrates. A decagonal phase with the tenfold axis $\mathrm{A}_{10}$ parallel to substrate surface normal was observed with two different unique orientations, which were related by $6^{\circ}$ rotation along the tenfold axis with respect to each other. The two orientations are $\mathrm{A}_{2 \mathrm{P}} / /[10 \overline{1} 0]$ and $\mathrm{A}_{2 \mathrm{D}} / /[10 \overline{1} 0]$ of the substrate. Using the extension of the CRLP model for quasicrystal-crystal epitaxy, we are able to determine the minimum energy for the interface and hence to predict and explain the stable geometric relationship between quasicrystal-crystal interfaces.

\section{ACKNOWLEDGEMENTS}

This work was supported by the US Air Force Office of Scientific ResearchDepartment of Defense award F49620-96-0214. The authors would like to thank Technology Assessment and Transfer, Inc. for providing the sputtering target, Dr Yimei Zhu for letting us use the JEOL-3010 at Brookhaven National Laboratory 
and Dr Wharton Sinclair for providing the C code to interface Digital Micrograph and Semper.

\section{REFERENCES}

Archambault, P., and Janot, C., 1997, Mater. Res. Soc. Bull., 22(11), 48.

Besser, M. F., and Eisenhammer, T., 1997, Mater. Res. Soc. Bull., 22(11), 59.

Bolliger, B., Dmitrienko, V. E., Erbudak, M., Luscher, R., Nissen, H. U., and Kortan, A. R., 2001, Phys. Rev. B, 63, 052203.

Collazo-Davila, C., Landree, E., Grozea, D., Jayaram, G., Plass, R., Stair, P. C., and Marks, L. D., 1995, J. Microsc. Soc. Am., 1, 267.

Dubois, J. M., 2000, Mater. Sci. Engng, A294-A296, 4.

Fletcher, N. H., and Adamson, P. L., 1966, Phil. Mag., 14, 99.

Fletcher, N. H., and Lodge, K. W., 1975, Epitaxial Growth, Part B, edited by J. W. Matthews (New York: Academic Press), chapter 7, pp. 529-557.

Giacovazzo, C., 1998, Direct Phasing in Crystallography (Oxford University Press), pp. 2, $18-20$.

Grimmer, H., Bonnet, B., Lartigue, S., and Priester, L., 1984, Acta crystallogr. A, 40, 108.

Grimmer, H., 1989, Acta crystallogr. A, 45, 320.

Ikuhara, Y., and Pirouz, P., 1996, Mater. Sci. Forum, 207, 121.

KIm, Y., and Hsu, T., 1991, Surf. Sci., 258, 131.

Li, G., Zhang, D., Jiang, H., Lai, W., LiU, W., and Wang, Y., 1997, Appl. Phys. Lett., 71, 897.

Jenks, C. J., and Thiel, P. A., 1997, Mater. Res. Soc. Bull., 22(11), 55; 1998, Langmuir, 14, 1392.

Shen, Z., Kramer, M. J., Jenks, C. J., Goldman, A. I, Lograsso, T., Delaney, D., Heinzig, M., Raberg, W., and Thiel, P. A., 1998, Phys. Rev. B, 58, 9961.

Shimoda, M., Sato, T. J., Tsai, A. P., and Guo, J. Q., 2000, Phys. Rev. B, 62, 11288.

Stemmer, S., Pirouz, P., and Ikuhara, Y., 1996, Phys. Rev. Lett., 77, 1797.

Susnitzky, D. W., and Carter, C. B., 1992, J. Am. Ceram. Soc., 75, 2463.

Sutton, A. P., 1992, Prog. Mater. Sci., 36, 167.

Sutton, A. P., and Balluffi, R. W., 1995, Interfaces in Crystalline Materials (Oxford: Clarendon).

Urban, K., Feuerbacher, M., and Wollgarten, M., 1997, Mater. Res. Soc. Bull., 22(11), 65.

Widjaja, E. J., and Marks, L. D., 2002, Thin Solid Films, 420-421C, 289.

Wolf, D., and YIP, S., 1992, Material Interfaces (London: Chapman \& Hall).

Xu, P., JaYaram, G., and Marks, L. D., 1994, Ultramicroscopy Lett., 52, 15.

Zhang, J. P., Cheetam, A. K., Sun, K., Wu, J. S., Kuo, K. H, Shi, J., and Awschalom, D. D., 1997, Appl. Phys. Lett., 71, 143.

Zhang, Z., and Geng, W., 1992, Phil. Mag. Lett., 65, 211.

Zhuang, Y., Zhang, Z., and Williams, D. B., 1993, J. non-crystalline Solids, 153-154, 119. 